\title{
Capturing User Access Patterns in the Web for Data Mining
}

\author{
I-Yuan Lin, Xin-Mao Huang, and Ming-Syan Chen \\ Electrical Engineering Department \\ National Taiwan University \\ Taipei, Taiwan, ROC \\ Email: mschen@cc.ee.ntu.edu.tw
}

\begin{abstract}
Existing methods for knowledge discovery in the Web are mostly server-oriented and approaches taken are affected by the use of proxy servers. As a result, it is difficult to capture individual Web user behavior from the current log mechanism. As an effort to remedy this problem, we develop in this paper methods for design and implementation of an access pattern collection server to conduct data mining in the Web. We also devise an innovative method, called page conversion, which converts the original Web pages to enciphered ones so that the devised data collection mechanism will not be deliberately bypassed. With the concept of page conversion, the methods we proposed involves a mechanism of software downloading to resolve the difficulty imposed by proxy servers and to effectively capture the Web user behavior. Using the devised mechanism, traversal patterns are generated and compared to those produced by the ordinary Web servers to validate our results. It is shown that the traversal patterns resulting from the devised system are not only more informative but also more accurate than those generated by ordinary Web servers, showing the importance and the usefulness of the mechanism devised.
\end{abstract}

\section{INTRODUCTION}

Capa t ur ur i ngl\$ or M eI r c apa ahhUPf appLgr PhAaPgUhU pIb - Uhet Uar it f egpar p gUtaghA agla - UhaoPUes pAUu hgtaPr i oPU es het fopri seg WgeoP af f Dhaper P Y, X Hd S r al bPP es f aPp pgar Pahpler capa har f geWc U Wgb WDy a- ID ur segt aper er hoPpet Ug - obu i - UAaWegn ar c pAoP t f geWU pAU Eoalpb es - oPr UP c UhPer Pd 1 agu eoPcapa $t$ ur u haf a- upulPAaW- Ur Unf Ig gte u pAU Iptgapogthur hDc ur i aPfehaper goIDP Y, XYT,nhIAPPy whaper goIDP KX,nPEoU puDf applgr PY, IL, nar c R U capa t u u i YY, Ya, $\mathbf{~}, \mathbf{L O}$ Y: , YNnpe rat Ua sU d

Aeoi A gUB PP es c uflgup pef AlPphaper IDWUP- Uy ur i f geWc Uent ePp t LpAecP seg Mr eI Dei UcufheWgb u pAUR U AaWpAUgLPghpler P pAap pAUb agUt ePID PgWgyegur ple ar c I LgUr ep Unf Dhuplb c URi r le seg Ly sUhpuW hDUt py aPle capa t ur i (uth haf pogur i r cuy Wc oaDR U oPg - UAaWeg)d Ur c LgPar c ur i ur c uhc oaD oPg - UAaWeg PoPsoDr t ar b aff UhpPrur hDc u i - Чy plgf UgPer alozle PgWhthut f geWte R U f ai Uegi ar vay

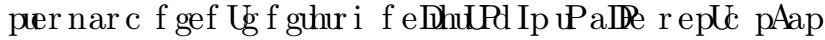
f geg I egMer R U capa t ur u I lgUPet U Aap Dt y ule coUpe pAUf gLPU hUes f genb PgWgP u pAUR U

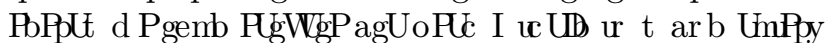
ur i R U RpLPseg Uffihul hb ar c Phogupb gLaPer P Y0,d S Pf eur ple eop ur K,ncoUpe pAUoHes f genb PgWgPh R U PgWgPI uDer ID gUhegc pAUahhUPes pAUPUf genb PgWgPar c agUr epa- IDpe - Uc upur i ouPApAUr Ur pupuP es ur c uhc oaDoP@P- UAur c pAUf genb HgWgd S P PohAn - b ar aIDzur i pAUPU capa Đi Pher Uhar r ep haf pogU ur y c unc oaDoHg - UAaWegd Cer PEo Ur plDn UfthpuWub haf y pogur i oPg ahhUPf applgr Pu pAUR $U$ seg capa t ur u i PpAUe- jUhpuWUes pAiPf af Łgd

AUt LAecPI UcUWFe agU-aPt er pAUher hUf p es f ai Uher WgRer ar c t au ID oPl seg U Wgert U pP I AlgU pAU f gulhb seg oPgP - UAaWeg ur pAUR U $\mathrm{P}$ rep a herhढgr arc pAUr Ute seg capa t ur u i PjoPpy wte (eg capa heIDohper PAeold r ep-Ucer Uu pAUwgPp $\mathrm{f}$ ahU)d . AU t UhAar ut es eog af $\mathrm{f}$ geahA u WLWP a Pespl agUceI r Dacur i ar c I egM aP seIDI Pd Fyppn a R U f ai UPU hec U ur pe ho AUg - b a PgWgyre UUy huf Algur i t ecoIDd. AUegu ur aDR U f ai Uugtf lahte - b pAuPU huf Algle R U f ai Ud. AuPuPhaIded f ai Uher y WgRerd. AUr na hUlr p Re Uf gei gat nhaIDoe c Uhu Algy

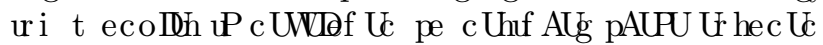
capa ar c pe cuff ab pAU her pUp pe R $U$ oPgPd . AU c Uhu Algur i t ecoIDuPpAUPespI agUpe - UceI r lac le sget pAUPgWg pe pAUhWurpd. AUc Uhr Algur i t ecoID I UDaIDe gIf egp pAUoPg - UAaWég pe pAUahhUPf applgr heIDLhpuer PgWtg (S PCS) - LsegUpAUcapa Pc Uhu ALg屯 ar c PAeI rd Bb AaWri pAU Ur huf Alguri ar c c Uhu Algy u i t UhAar ut ner Uhar U PogUpAap pALPUR $U$ f ai UP I uDr ep - U PAeI $r$ or IDPP pAU c Uhf Algur i t ecoID $\mathrm{P}$ haIDoc ar c pAUS PCS Pur segt UenPe pAappAUcapa hely IDhper f gehUPI uDrep - Uc UD LgapUD - bf aHted (Os heogPhpAUtr huf Algur i /c Uhu Algur i t UhAar ut har - U glt eWte is pAUPBPte aIDI PoHgPpe - bf aPPpAUcapa heIDhpher f gehUP(). I e c Uhu Algu i t ecoIDPngUf Uhy puWUB c UPi r Le seg NLphaf Uar c MSIE - geI PgPhagU

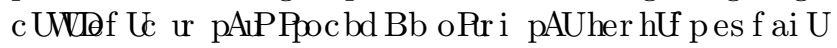
her WgRer npAUt UpAecPI Uf gef ePE gLPDWpAUc uffiy 
ho Pb it $f$ ePte - $b$ f genb PgWgPar c Ufthpulab haf pogU pAU R U - UAaWegd R pA oHg ahhUPf f applgr Pf gef y UgID heIDChplen pAU ur c uhc oaDR U oPg - UAaWeg har - U- Upplg haf pogle ar c ar albzle - b pAUheggLff er c u i capa t ur u i pLhAr woLPd

AlPf af Lg Pegi ar wL aPseIDI Pd. AUt LpAecPI U f gef ePt pe heIDohp oHg ahhUPf $f$ applgr P u pAU R U agUc Uthot Ute ur SUhper vd S f f thaper Pes S PCS agU f gUPF ple ur SUnper Od. AU ac War pai U es It f labur i pAUßi Psget pAUS PCS seg t u u i pgaWgraDf applgr P P WIDe aple ur SUhper : d. AlPf af Lg her hDc UPI pA SUhper Xd

\section{N TRøu CUI ImpltmtutCt@U OF CU APCS}

Ir pAiPPhpier nI UPAaDb UAngu UpAUc URi $r$ ar c it y f IDt Ut paper es ar ahhUPP f applgr heIDDhper Fglogn

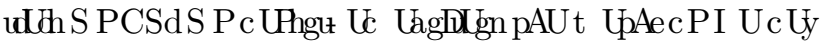
WFe agU-aPe er pAU her hUf $p$ es $f$ ai $U$ her WgRer d Cer hUf poaIldon up paMP wW Ppuf P seg a oPd pe - geI PU pAU her plip es pALPU U huf Algle f ai UPd FuPpn a oPt ef UPPAUR $U$ f ai U- b a R U - geI Pønar c pAUR $U$ - geI Pi Pf cPar H. . P gLEoLPp pe pAUR U PgWg seg

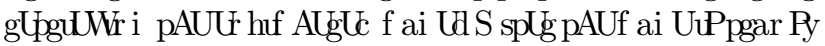
slggle sget pAUR U PgWtg pe pAUR U - geI Pøn pAU - geI P\& $\mathrm{PaI}$ agUpAap pAUf ai UPAeod - Uc Uhuf Algle ar c PIr cPpAUherpurp pe a c Uhuf Algur i t ecoIdd. AU

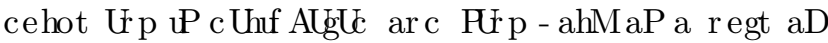
H. ML segt ap pe pAU-geI Pgnar c ap pAUPat Upt Un pAU c Uhr Algur i t ecoIDr epuwP pAUS PCS a- eop pAU oPg ahhUPpe pAtPc ehot Uf pd Fur alDhnpAUR U - geI PG

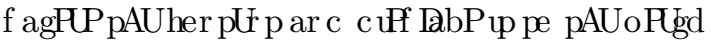

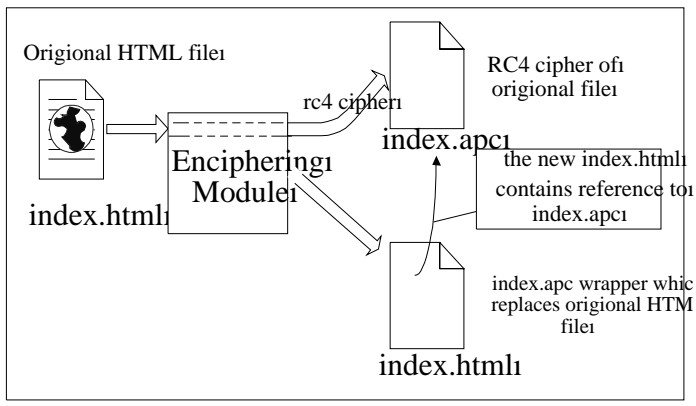

Fú ogUL: Er huf Alguri ar c I gaf f u i

AgUhet f er U pP AaW - Ur c UWJef Len Unf ThuplB (L) pAUU huf Algur i t ecoIhh(v) pAUS PCSnar c (O) pAU c Uhu Algur i t ecoIdd. AUwgPp e het $f$ er U pPagUgor

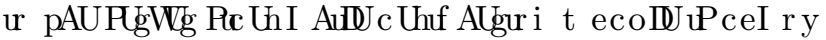
Dac Le pe pAUhHur p Re Uar c gor pALgLd NepUpAap pAU

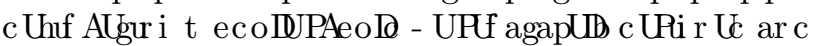

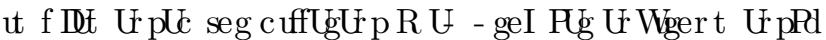
Oog c Uhr Algur i t ecoIDAaP- Ur ut f IDt Ur ple er pI e t ajeg R U - geI PgRudhNaWi apeg ar c MSIEd

S PPAeI $r$ u Fú ogULnpAULu huf Algur i t ecoIDuPgu Pf er Pt ID seg pI e paPMPd Or U P U huf Algur i ar c pAU
epAlg u I gaf f u i d GuNk ar H. ML wWJ (I pA dApt D

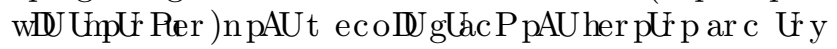
huf AlgP pp oPr i RC: aliegupAt I AthA PoHe I upAeop

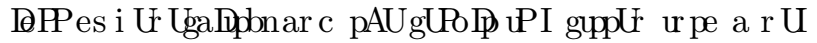
wDI upA daf $\mathrm{h}$ wDU Unpli Rer d

S splg pAU U huf Algur i f gehUHh pAU egi u aDH. ML wDJPher Wople pe a r U H. ML wDhhaIDde a I gaf $\mathrm{f}$ Lg H. ML wdid. AUI gaf $f$ Lg H. ML her paur PpAgUhet y f er Uphudth(L) pAUhec Upe ur WMc Uhr Algur i t ecy

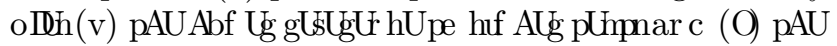
hec Upe c uff lab pAUher pUp p u pAUR $U$ - geI Pogd

Feg NLphaf UNaWi apegn pAUc Uhu Algur i t ecoIDuP I gupd广 aPa f Di yrd. AlglegthpAUI gaf $f$ Lg wldher y RAPPes ar H. ML pai (<Ca pt u r >i ng Unse AcPhWhigh bfoDkE - UAsDuncYLsff, cLefcU, ngh cXIthdn, UnU Le, blcAs, h ngh dcenhen nc ngh bfoDke S c, ofhI - cnh ng Un ngsWfoDke S c, ofh Lsff e cncefy, hdsbghYngh, UnUXon UfW dcffhdn ngh o WYseECIS Unsce Le, We, sn XUIP nc ngh 1 mu N 1 FhhYngh, hdsbghlseDb Ic dhWWngh dcenhen sW, sWffuh, nc ngh o WYXy UpUAUNd)sbn IconsehI

Ce ngh cnghYgle, Tngh, hdsbghlseD Le, , sWfuyM seD bledhWLc PPWAS sfUry se w Ntt Lsng WS h, sEM Hhithedh se sS bfhS henUnsceI $\mathrm{r}$ ghth sWle $<$ Capt ur $>$ nUDse L YubbhYRr w O : fh nc hS Xh, Ue 1 dnsAh@ dceM nlefI w Nit Lsff , cLefcU, ngh l dnsAh@ dcenlcfTse Wuff Le, h. hdonh snI

III. N TTRDuCa MP I L UM N T OF CI AuCu P OMOMC

G ptuar i ng Us eAcPauts hePAc we PAlett Wor Abattot bf DMA I P-othulKPAlett os ehpte- aPs ehc bto aPs ehcbto PLL-ett PaL oce, XCbf oce Hed I Pre PAS AetteLYg bs I P-eL ob Wort a Pa b-LiaP-y Hed te-Me-h i ng UWr APa r ule PLeEpPoe uafb-s Poba fb- pte- LeaS owDAPobaYl cpthbae APa PI I LLLPOPs la ar oeAcawpet ob ocete i ng U Wirt PaL reo I e-tbaPtheL LPoPs calar -etplatY

6 Nunde teea $\propto$ Po oce pte bf i ng U ceVt dboc te-M-SiPteL PaL AlteaosiPteL LPoP s wararY Ue-Me-S dPteL LPoPs a la ar - efe-t ob oce APte oc Po r ulfa P H ed te-Me-h bae it ob LitAble- oce TabNuLre Pdbpo NcPo P-e oce pte- decPMb-t bf oc bte pte-t Mttorar oc t H ed tweYG Abao-PAbhAlheastPteL LPoPs wa lar -efe-t ob oce APte oc Po r ulfa P pte-h bae NbpW tate ob TabN NcPo P-e oce Ned twet oc ut I P-oupla- pte- Mt ot Y wb- ulWtS o-Pould I p-I btethbae t AeaP- b it I-eteaoL u wr p-e v fb- AldeaosiPteL LPoPs wa tarY 


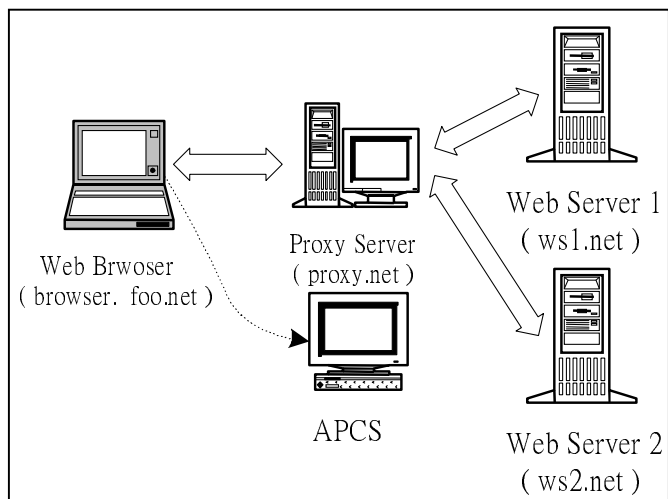

Capt ur i n gUrs euaAcAu Uars hWefro oehe Das as $\mathrm{p}$

MAs faruhI r fUss euaAs Capt ur i - YI rur e L rb t fru af eUrff $\infty \mathrm{p} \mathrm{L} \mathrm{rb}$, eprf क hYAL rb fruXrufHd I r , uASy fruXru t fro by $h I$ of $t$ fru of , uASyHrhH Fe hI r t fru eUW Ufffrf As r , epr क L rb fruXru l- cAPAYro by es AhI ru , epr eUrff hAL rb fruXrui - es o fAcAuhI - Yr I eXr a W orSl \#hDPas L rb fruXrul - a orSl \#hDPas L rb fruXru i - ж orSi HlhDPas L rb fruXrul es o ж orSi HhDPas L rb fruXrui - ef as oalehro brPAY - urft Pras $\mathrm{p}$ \& hYAPApf hI eh eur Xruy oamU Pl hAUADbar r es o es ePNH

TUrff PAp aAD L rb gruXrul

, uASyH rhlw Rd ж orSl HhDPOd d : @ HwW

, uASyHrhlw Rd क orSi HhDPOd d : a HwW

TUrff PAp aAD L rb gruXrui

, uASyHrhw Rd क orSl HhDPOd d : G HwW

, uASyHrhut Rd क orSi HhDPOd d : a HwW

ff $\mathrm{s}$ hI $\mathrm{r}$ AhI ru I eso- hIr PApf क L rb fruXruf eso

T: Mg eur fI AYs ef brPAYH

TUrff PAp aAD T: Mg

browser.net- "GET http://ws1.net/index1.apc HTTP/1.0"-

browser.net- "GET http://ws2.net/index1.apc HTTP/1.0"-

browser.net- "GET http://ws1.net/index2.apc HTTP/1.0"browser.net- "GET http://ws2.net/index2.apc HTTP/1.0"-

( y es eRNos $\mathrm{p}$ : Mg eUrff PApf-As $\mathrm{r}$ Les UAurUhP hueU hIr tfru eUrff, ehIf eUAAf stDruAtf L rb fruXruf- hI tf ceLaPhehs $\mathrm{p} \mathrm{hI} \mathrm{r} \mathrm{YAu)} \mathrm{cAu} \mathrm{UPrs} \mathrm{hWefro} \mathrm{oehe}$ D क ж pHUAhr hI eh hAft DDeuar t frueUrff , ehI f by es eRM $p$ eUrff PApf of hubt hro eUuAf ozrurs h AuoaW s euy L rb fruXruf- hI r haDru As hI rfr L rb fruXruf I eXr hA br fys U uAs aNoHMAs ot Uhas $\mathrm{p} L \mathrm{rb}$ oehe Das as $\mathrm{p}$ by es ePy $\mathrm{p}$ T: Mg eUfff PApf, uAXarf e crefabP fAP W haAs hA hI of , uAbPDH RS, PatahP- ef YaPbr XePoehro brPAY- hI r eUrff PApf Abheжs ro by hI r T: Mg YaP, uAW Xaor eorI t ehr a cAuDehaAs cAu L rb oehe Das a pH

\section{N T R ODUCIMP L TO F APC SUPS FUR TRAVORSAD AAL L ORM DORIVAL IUM}

Cap turinutg Utsnet Arct p Phe Pg WurPbt ns Cf o D aMartp ia ira IP- PUirMns nUrPiuiub Late igturirita, $\mathrm{XaH}$ iub rct Cf o D YbaAd t IPu tSrePIr Pug InuareLIr rct rePUeaPY- Prrteua sne iugiWgLPYLatea, y nrt rcPr Yba senp rct negiuPeME tUateWea IPu Płn Ut Latg rn btuH tePrt rePWeaPY- Prrteua lnN, Tnd tWeAiu Pu negiuPeM E tUateWeArct Late Utc PWhea Pet Ybbtg iu Pu iurteH YPUtg p PuuteAPug rct iusnep Prinu ns Late igturirMia unr iuIYgtg iu rct Yb, Ca P etaLYArct - Prteua gtH eiWg senp rct negiuPeME tU ateWe Kb IPu Ut tircte iuInp - Yrt ne iuIneetIrArcLa LarisMub rct utItaairM ns Laiub rct Cf o D ateWe,

\section{A. Mining on Logs from Ordinary Web Servers}

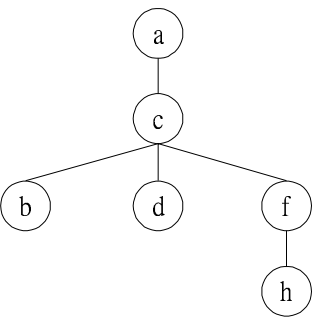

Capt ur ing UrseAcP h rWbefr oDMh rWceprbI

\begin{tabular}{|c|c|}
\hline Users & Traversal pattern \\
\hline User 1 & $(a, 1)(c, 4)(a, 6)(c, 9)(d, 11)(c, 12)(f, 15)(h, 17)(b, 18)(c, 19)(f, 20)$ \\
\hline User 2 & $(b, 2)(c, 3),(d, 5)(c, 7)(f, 8)(h, 10)(f, 13)(c, 14)(a, 16)$ \\
\hline
\end{tabular}

- eWP Yn L brue, ,rbb brXtrU, r

HoUbedru fSr rseAcP h rWbafr aU Capt ur i y SrurE $y$ a Sot $f$ Bbb oDprUruePflEm Uodrb eur WaUp Nabafrd W T t brub y So eur ebbt Ard fo po fSuot pS fSr beAr cuosl bruNu fo e, , rbbfSab h rWbafrI - Sr , ourbcoUdw aUp fueNubePbr Xt rU, rb W fSr Tt brubeur paNU aU - ew WP Y - Srur@urEe, oudaUp fo - eWr YEe brXtrU,r oD fSr e, ,rbb Bp §u fSr fueN.PaUp oDfSrbr t brub aU eU oudaUteul h rWbrulvu ab eb D\#8y n

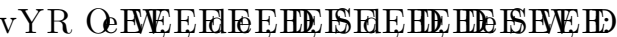

@ofr fSef baU, r fSrbr T t brub eur WrSaUd fSr beAr cuosl bruNuEy r y aPUf W eWP fo dabfaUpt abS fSr fueNubePur, oudbaUfSab Pp DoA oUr t brufo fSr of SruI HoUbrXtr IfPEePourSA . C aU ffin , eU W r Ac Bl rd fo rsfue, f fSr Aes aAePDuy eud ur DurU, r e, , oudaUp fo fSrbr fueNubePur, oudbI - SrUEt batp epoufSA . CE fSr , ourbcoUdaUp brf oDA es aA ePDuy eud urDurU, rb , eU W oWeaUrdI Cou rseAcPEy of fSr NePr oDfSr A alaAePbt c c ouf WaUp TeUd fSr AaUaAeP, oU) drU, r WaUp LzI EeAoUp ofSrubEfy o ceffruUbEOW $\mathrm{R}<\mathrm{Q}$ : eUd $\mathrm{Q}$ : $\mathrm{R}<\mathrm{O}$, , eU W druaNdI OW $\mathrm{R}<\mathrm{Q}$ : AreUb fSef rNul Nabof fo Wy aPW DHOyrd W e Nabef fo, I @ofr fSr Ut A WruoDo, , t ur U, rboDOW ab TeUd fSef oD OW: ab ePo TEurbt PaUp aUe , oU) drU, r oDIPTR YzzI DufSr fueNrubePceffruUOW $\mathrm{R}<\mathrm{Q}$ : I ffiteddafaUEy of fSr Ut A Wu oDo, , t urU, rb oDQ: WaUp 0 eUd fSef oD Q D WaUp CEO : R < CD AreUbfSef CPO R UOI oDfSr Nbbefb fo, y alPW aA ArdæfrP DPOyrd W Nabfb fo ID 


\section{A. Mining on Logs from Od a y}

G pt auri nuguUs nse Asaps tci ppsnn Rh crt WuUs uri bf sPlah tc ulsns D Ansrn ca i a MI - YontLui cas, i pptr, f cah ut Xi LB Hi not Pld nS

$\mathrm{S} 2=\{(\mathrm{a}, \mathrm{U} 1)(\mathrm{b}, \mathrm{U} 2)(\mathrm{c}, \mathrm{U} 2)(\mathrm{c}, \mathrm{U} 1)(\mathrm{d}, \mathrm{U} 2)(\mathrm{a}, \mathrm{U} 1)(\mathrm{c}, \mathrm{U} 2)(\mathrm{f}, \mathrm{U} 2)$

$(\mathrm{c}, \mathrm{U} 1)(\mathrm{h}, \mathrm{U} 2)(\mathrm{d}, \mathrm{U} 1)(\mathrm{c}, \mathrm{U} 1)(\mathrm{f}, \mathrm{U} 2)(\mathrm{c}, \mathrm{U} 2)(\mathrm{f}, \mathrm{U} 1)(\mathrm{a}, \mathrm{U} 2)(\mathrm{h}, \mathrm{U} 1)$

$(\mathrm{b}, \mathrm{U} 1)(\mathrm{c}, \mathrm{U} 1)(\mathrm{f}, \mathrm{U} 1)\}$

Captp u rting « eAc P pus h raur Wht Abihirh s f op s D

Mf s hpI Wós rge Cp Yus aubp is oibioWgg P f bis L hp,

I Wos Yph Xf P HdMS of Lh uh is y uFgp l D

mhpt 1 NTue Ye ue Yé oe Ye Xae Ee Ye Xv

mhpt vNTEe Ye oe Ye X ae Xe uw

Mf s hpI Was rge rap gutLp irpP hprh Yus Ep f Eruis poD Ris ugo e Cira rap huP p If s Oops Yp ulple : @ c us o rap $\mathrm{P}$ is iP ug hWnf tr LTpe vce $\mathrm{uP}$ f s L f rapthe ratpp nur, rpts he TEw ff $<$ Capt Cp r $<$ G pt ngU Caup $\mathrm{r}<\mathrm{C}$ pt ang s e UeAPeU naahAlog Wfo DhMd NinMCaup $\mathrm{r}<\mathrm{G}$ p d ns IegMułh- Nile AIYbII UeAPeU sf Nile hALgnAf L es IeAPeAhW, Wht UeIXald hus eg Whs be Ni WgeAnld Ih- e ahAleaMXnNAAgI rete Cs p r < Capt NiIe bWuA- ng hALod gnAf L es IeAPeA- nf ænU N cgahAleaMXnNAAgIt IYai nI Cap r < Cpt S i cai t nI ang s e PeAcyeUsf Nile hAckd gnbMAPeAnbIeEYegae cg 1 ns be nt d n s hWI hge Noect S dilhYMeghYW ahgyUegaeTo whang s e Ieeg NiInMIYai XAhs k- I nłe nPhdeUS i eg Nile bWü- Nile, vROd e- Xbhf eUb

\section{I . N TRODUCITR}

Ca ptur i ng User sUAn ec API User hUtuer bng Uf o DM I UAWAetU WteKU ngU-Usp Ya u AetU Xa AWњ tUWUW Pr - UALnhUa sPAnpW ng US UX ptUWXUPhy weVEl turi ngU-UhtU, Lsgr it, bnWhUAPYAPnnUtt gPhUXUr i U UPhU Pr - se, APW ne ngetUAW-psU Xa ngUeWur PLA S UX tUhUW ne hPY PnUepWwtpYitEn I Pt tgeI r ngPn ng UnWhUAPYAPnnUwt WtpYri i cld, ng U- Uhut tatnU, PWr en er F , eWur œW PnhUXpn PYe , eWPsspWhUngPr ngetUi U UPnU Xa eWu PX S UX tUWUAbtgeI ui ngUu AeMPrsUPr- ngUptLepY r Utt ec ng U, $\lesssim g P r$ t, - Uhit E

\section{Acknowledgements}

Cap turai ng tmp guUUi nrps eA Ut nr cP rap h tre At W bf epAfp o i uAf eWM Mi Ipf $\mathrm{r}$ h i - h bo Y, XXH, d, SSX SSy t As h bo YL, XXHE, d, SSX,SEXDCted t ADmpUuc i N o aeAt -

\section{I . N. T. RO. D}

[1] R. Agrawal, T. Imielinski, and A. Swami. Mining Associations between Sets of Items in Massive Databases. In Proceeding of ACM SIGMOD, pages 207-216, May 1993.

[2] R. Agrawal and R. Srikant. Mining Sequential Patterns. Proceedings of the 11th International Conference on Data Engineering, pages 3-14, March 1995.
[3] G. O. Arocena and A. O. Mendelzon. WebOQL: Restructuring Documents, Databases and Webs. Proceedings of the 14th International Conference on Data Engineering, pages 24-33, February 1998.

[4] A. G. Buchner and M. D. Mulvenna. Discovering Internet Marketing Intelligence through Online Analytical Web Usage Mining. ACM SIGMOD Record, 27(4):54-61, December 1998.

[5] M.-S. Chen, J. Han, and P. S. Yu. Data Mining: An Overview from a Database Perspective. IEEE Transactions on Knowledge and Data Engineering, 8(6):866-833, 1996.

[6] M.-S. Chen, J.-S. Park, and P. S. Yu. Efficient Data Mining for Path Traversal Patterns. IEEE Transactions on Knowledge and Data Engineering, 10(2):209-221, April 1998.

[7] D. W. Cheung, V. T. Ng, W. Fu, and Y. Fu. Efficient Mining Association Rules in Distributed Databases. IEEE Transactions on Knowledge and Data Engineering, 8(6):911-922, December 1996

[8] R. Cooley, B. Mobasher, and J. Srivastava. Web Mining: Information and Pattern Discovery on the World Wide Web. IEEE Conf. on Tools with Artificial Intelligence, pages 558-567, 1997.

[9] R. Cooley, B. Mobasher, and J. Srivastava. Data Preparation for Mining World Wide Web Browsing Patterns. Knowledge and Information Systems, 1(1), February 1999.

[10] R. Fielding, J. Gettys, H. Frystyk, and T. Berners-Lee. Hypertext Transfer Protocol - HTTP/1.1. Technical Report Request for Comments: 2068 Internet Engineering Task Force, January 1997.

[11] J. Han, G. Dong, and Y. Yin. Efficient Mining of Partial Periodic Patterns in Time Series Database. In Proceeding of the 15th International Conference on Data Engineering, pages 106-115, March 1999.

[12] D. Konopnicki and O. Shmueli. Information Gathering in the WWW: The W3QL Query Language and the W3QS system. ACM Transactions on Database Systems, December 1998.

[13] L. Lakshmanan, F. Sadri, and I. Subramanian. A Declarative Language for Querying and Restructuring the Web. Proc. 6th Int. Workshop on Research Issues in Data Engineering, pages $12-21,1996$.

[14] M.Spiliopoulou and L. C. Faulstich. WUM: A Web Utilization Miner. Proc. of EDBT Workshop WebDB98, 1998.

[15] R. Ng and J. Han. Efficient and Effective Clustering Methods for Spatial Data Mining. Proceedings of the 18th International Conference on Very Large Data Bases, pages 144-155, September 1994.

[16] J.-S. Park, M.-S. Chen, and P. S. Yu. Using a Hash-Based Method with Transaction Trimming for Mining Association Rules. IEEE Transactions on Knowledge and Data Engineering, 9(5):813-825, October 1997.

[17] S. Schechter, M. Krishnan, and M. D. Smith. Using path profiles to predict HTTP requests. Proceedings of the 7th International World Wide Web Conference, 1998.

O. Zaiane and J. Han. WebML: Querying the World-Wide Web for Resources and Knowledge. Proc. (CIKM'g8) Int'l Workshop on Web Information and Data Management (WIDM'98), November 1998. 